\title{
Migraine management
}

\section{Bronwyn Jenkins}

Neurologist, North Shore

Vertigo and Neurology

Clinic, and Royal North

Shore Hospital, Sydney

\section{Keywords}

calcitonin gene-related peptide, migraine, triptans

Aust Prescr 2020;43:148-51

https://doi.org/10.18773/ austprescr.2020.047

\section{SUMMARY}

Migraine causes significant lost time from everyday activities. Addressing lifestyle triggers and comorbidities in patients with migraine is the first step of management.

Acute migraine treatments primarily manage the headache component and should be started as early as possible in the migraine attack.

Prophylaxis may be recommended if a patient is having three or more migraines a month or if their migraines are difficult to manage.

The choice of prophylactic drugs should be tailored to the individual's potential for adverse effects, interactions and comorbidities.

\section{Introduction}

Migraine is more than just a headache - it has associated features including sensitivity to light or noise, nausea and avoidance of exertion. The headache is typically throbbing and severe. As such, migraine is debilitating and is the leading cause of disability in people under 50 years old.

\section{Pathophysiology}

Migraine is a common, polygenetic brain disorder with complex biology. The vascular reactivity of aura is now considered to be a secondary symptom that occurs alongside migraine. There is convergence of peripheral trigeminal sensory nerves on the single pain centre of the trigeminocervical complex. The central mechanisms include neurotransmitter pathways such as serotonin, calcitonin gene-related peptide and other neuropeptides. ${ }^{2}$

\section{Assessing the patient}

To distinguish migraine from other causes of headache, ask the patient about the character and location of the headache as well as associated features and avoidance of exertion. Differentiating between episodic and chronic migraine is an arbitrary but useful cut off for some treatment options (see Fig.). In clinical practice, patients highlight their worst migraines. To ascertain the true frequency of headaches, enquire about the number of completely headache-free days per week or month.

\section{Management approach}

Addressing lifestyle triggers and comorbidities in patients with migraine can be particularly beneficial for patients. Sleep disorders, dietary triggers (e.g. some types of alcohol, cheese, oranges and chocolate), dehydration and caffeine overuse are important to recognise and manage. Healthy body weight and exercise are recommended. Depending on the individual triggers, behavioural and psychological strategies and physical therapy can help some patients. Commonly used natural migraine preventives include magnesium, riboflavin, coenzyme Q-10, and Feverfew. These have limited and variable levels of evidence for efficacy and are not the mainstay of treatment. 3,4

\section{Pharmacotherapy}

Drugs can be used to treat acute migraine, or they can be used prophylactically to reduce the frequency and severity of attacks. The Therapeutic Guidelines has recently updated its guidance on headache, including general principles, specific dosing recommendations, and advice for children and pregnant women. ${ }^{5}$

\section{Treating acute migraine}

Treatments for acute migraine aim to abort the headache stage of migraine within 1-2 hours. Although effective for this, they do not significantly help with the prodromal, aura or postdromal stages. Treatment should be started as early as possible in the headache phase and some patients will require a combination of therapies. Common medicines used include paracetamol, aspirin (900-1000 mg per dose) or other non-steroidal anti-inflammatory drugs (NSAIDs), antiemetics and triptans. ${ }^{6,7}$

\section{Triptans}

As they are more selective against migraine, triptans ( $5 \mathrm{HT}_{1}$ agonists) may be first-line drugs for patients with moderate-severe pain, or when simple analgesics have not been effective. Triptans cause vasoconstriction and are not recommended in patients with cardiovascular disease. ${ }^{8}$ There are five triptans available in Australia. Guidelines recommend trying triptans sequentially to find the 
best tolerated and most effective option for the individual. Eletriptan, rizatriptan and zolmitriptan have the highest pain-free rates at two hours and naratriptan is associated with lower adverse effects (see Table). 2,9,10

Depression and anxiety are common comorbidities with migraine. The risk of serotonin syndrome when triptans are used in conjunction with selective serotonin reuptake inhibitors or serotonin and noradrenaline reuptake inhibitors is low. A recent retrospective data analysis showed there were only two confirmed cases of serotonergic syndrome in a cohort of 19,017 patients who were co-prescribed a triptan and an antidepressant."

Medicine overuse can worsen migraine. Triptans should therefore be limited to less than 10 days a month and simple analgesics to no more than 15 days a month. Opioids are not recommended for migraine due to limited effectiveness and the risk of drug overuse.

\section{Managing nausea}

Intercurrent nausea can impair absorption so taking an antiemetic with the first analgesic can help. ${ }^{12}$ If patients are unable to take oral medicines, other routes of administration can be considered:

- non-oral triptan formulations

- suppositories, such as NSAIDs (indometacin or diclofenac)

- ondansetron wafers for nausea and vomiting

- prochlorperazine suppositories.

\section{Fig. Principles of migraine management}

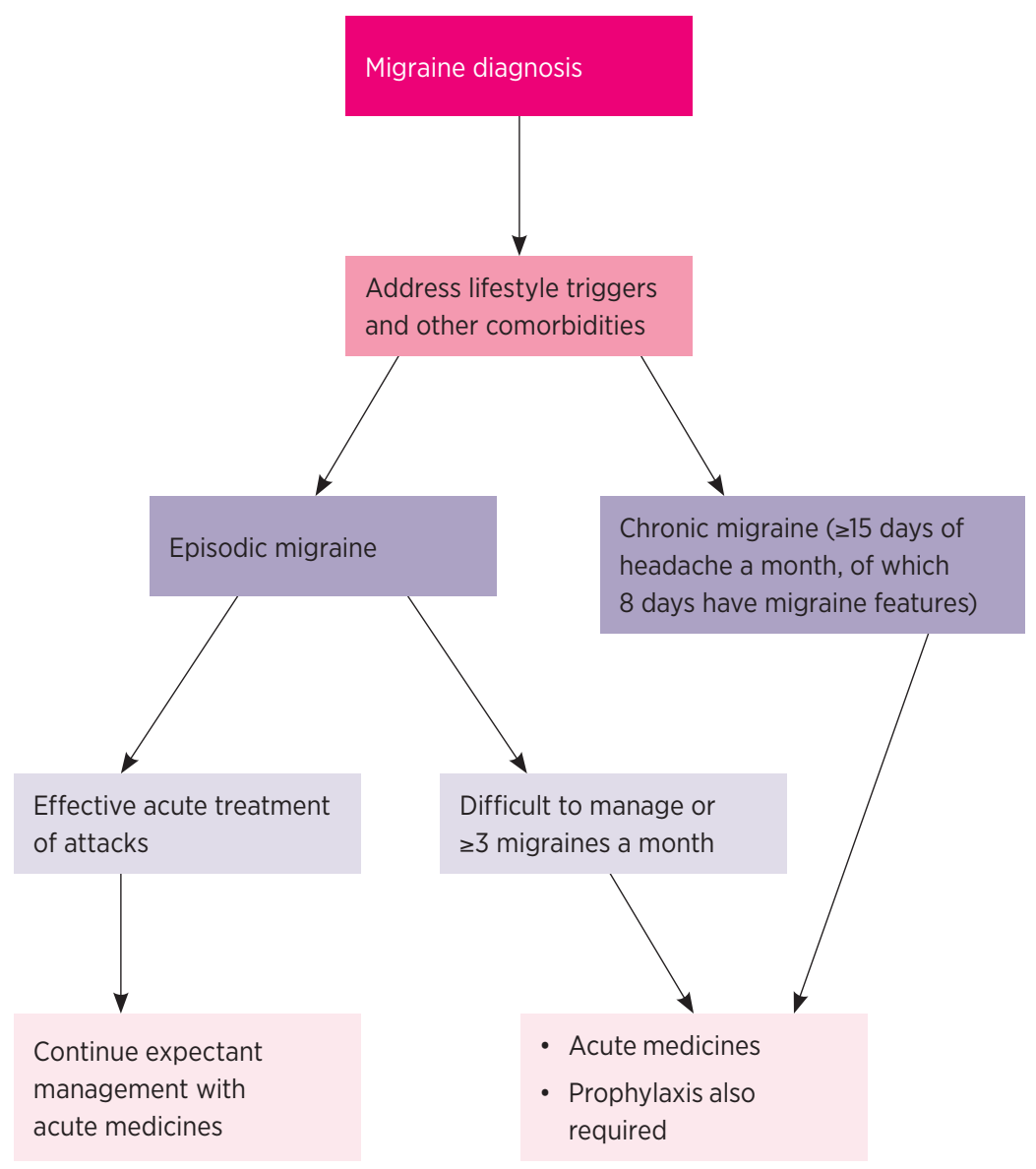

\section{Table Triptans available in Australia for migraine $e^{2,9,10}$}

\begin{tabular}{|c|c|c|c|c|c|}
\hline Generic name & Formulation & $\begin{array}{l}\text { Dosing } \\
\text { (maximum dose) }\end{array}$ & $\begin{array}{l}\text { Initial 2-hour } \\
\text { relief }\end{array}$ & $\begin{array}{l}\text { Sustained } \\
\text { pain free }\end{array}$ & Tolerability \\
\hline \multirow[t]{3}{*}{ Sumatriptan } & Tablet or fast disintegrating tablet & 50-100 mg (300 mg/day) & & & \\
\hline & Nasal spray (10 mg or 20 mg) & 10-20 mg one nostril (40 mg/day) & $=$ & $=$ & $=$ \\
\hline & Subcutaneous injection* & $6 \mathrm{mg}$ autoinjector (12 mg/day) & & & \\
\hline Rizatriptan & Tablet or wafer & 10 mg (30 mg/day) & + & + & $=$ \\
\hline \multirow[t]{2}{*}{ Eletriptan } & Tablet $40 \mathrm{mg}$ & & $=/+$ & $=/+$ & $=$ \\
\hline & Tablet 80 mg & & + & + & - \\
\hline Zolmitriptan & Tablet & $2.5-5 \mathrm{mg}(10 \mathrm{mg} / \mathrm{day})$ & $=$ & $=$ & $=$ \\
\hline Naratriptan & Tablet & 2.5 mg (5 mg/day) & - & - & ++ \\
\hline
\end{tabular}

* sumatriptan injection not subsidised on Pharmaceutical Benefits Scheme

Using $100 \mathrm{mg}$ sumatriptan as the comparator:

$=$ indicates no difference

+ indicates better

- indicates inferior, when compared with sumatriptan 


\section{Menstrual migraines}

Menstrually related migraine attacks are more severe, more difficult to treat and more likely to recur. A combined oral contraceptive pill can be used for up to six consecutive months to limit the number and choose the timing of the menstrually related attacks. However, the combined oral contraceptive pill should be avoided in migraine with aura due to the risk of stroke. In addition, some patients have increased migraine attacks on a combined contraceptive pill. Non-steroidal anti-inflammatories (such as naproxen) may help if there are any perimenstrual symptoms, in addition to the usual acute therapies. ${ }^{13}$

\section{Box Preventive treatments for migraine}

As first-line drugs in adults, use:

- amitriptyline* $10 \mathrm{mg}$ orally, once daily at night. Increase daily dose by $10 \mathrm{mg}$ at intervals of at least 1 week (maximum daily dose $75 \mathrm{mg}$ ). Continue at maximum tolerated dose for 8 to 12 weeks to assess efficacy

OR

- candesartan* $4 \mathrm{mg}$ orally, once daily. Increase daily dose by $4 \mathrm{mg}$ at intervals of at least 1 week (maximum daily dose $32 \mathrm{mg}$ ). Continue at maximum tolerated dose for 8 to 12 weeks to assess efficacy

OR

- nortriptyline* $10 \mathrm{mg}$ orally, once daily at night. Increase daily dose by $10 \mathrm{mg}$ at intervals of at least 1 week (maximum daily dose $75 \mathrm{mg}$ ). Continue at maximum tolerated dose for 8 to 12 weeks to assess efficacy

OR

- pizotifen $0.5 \mathrm{mg}$ orally, once daily at night. Increase daily dose by $0.5 \mathrm{mg}$ at intervals of at least 1 week (maximum daily dose 1.5 to $3 \mathrm{mg}$ ). Continue at maximum tolerated dose for 8 to 12 weeks to assess efficacy

$\mathrm{OR}$

- propranolol $20 \mathrm{mg}$ orally, once daily at night. Increase daily dose by $20 \mathrm{mg}$ at intervals of at least 1 week (maximum daily dose $160 \mathrm{mg}$ in 2 or 3 divided doses). Continue at maximum tolerated dose for 8 to 12 weeks to assess efficacy

OR

- sodium valproate*+ $200 \mathrm{mg}$ orally, once daily at night. Increase daily dose by $200 \mathrm{mg}$ at intervals of at least 1 week (maximum dose 500 mg twice daily). Continue at maximum tolerated dose for 8 to 12 weeks to assess efficacy

OR

- topiramate $25 \mathrm{mg}$ orally, once daily at night. Increase daily dose by $25 \mathrm{mg}$ at intervals of at least 1 week (maximum dose $100 \mathrm{mg}$ twice daily). Continue at maximum tolerated dose for 8 to 12 weeks to assess efficacy

OR

- verapamil* sustained-release $90 \mathrm{mg}$ orally, once daily. Increase daily dose slowly over 3 weeks (maximum daily dose $240 \mathrm{mg}$ ). Continue at maximum tolerated dose for 8 to 12 weeks to assess efficacy.

* At the time of writing, this drug is not approved by the Australian Therapeutic Goods Administration (TGA) for migraine prophylaxis. See the TGA website for current information www.tga.gov.au.

+ Avoid sodium valproate in females of childbearing potential (see eTG complete www.tg.org.au for information on teratogenic and neurodevelopmental effects of antiepileptic drugs).

See eTG complete for more detailed information on migraine prophylaxis www.tg.org.au Reproduced with permission from Migraine [Published 2017 Nov. Amended 2019 Jan]. In: eTG complete [digital]. Melbourne: Therapeutic Guidelines Limited; 2019 Dec. www.tg.org.au

\section{Migraine prophylaxis}

Prophylactic therapy ${ }^{14,15}$ is generally indicated in patients with:

- three or more severe headache days per month causing functional impairment that are not consistently responsive to acute treatments

- more than 6-8 headache days per month despite responsiveness to acute treatments

- contraindications to acute migraine treatments

- particularly disabling symptoms even if infrequent attacks (such as brainstem aura, hemiplegic migraine, syncope)

- ongoing significant impact to a patient's functioning despite lifestyle modifications, trigger management and use of acute treatments

- $\quad$ risk of drug overuse headache.

Considerations for choice of preventive medicines include evidence for efficacy, adverse effect profile, drug interactions, contraindications, patient comorbidities, costs, availability and patient preference.

All oral prophylactic drugs for migraine were developed for other purposes such as hypertension, depression and epilepsy. In general, they alter the neurotransmitters involved in migraine. Their efficacy can only be fully assessed after 8-12 weeks at a therapeutic dose.

Antihypertensives used for prophylaxis include calcium channel blockers (such as verapamil), beta blockers (such as propranolol), and angiotensin II receptor inhibitors (such as candesartan). Antidepressants include amitriptyline and nortriptyline. Antiepileptic drugs are also used topiramate is the most evidence-based of the oral migraine preventors, but carries potential adverse effects such as altered mood, verbal fluency issues (word finding) and paraesthesia. Sodium valproate is also prescribed as prophylaxis for migraines (see Box). ${ }^{5}$

Adverse effects and a patient's comorbidities often influence the choice of drug. For instance, medicines with a high risk of weight gain (e.g. pizotifen or sodium valproate) should be avoided in obese patients and beta blockers should be avoided in those with asthma. Antihypertensive drugs should not be given to people with hypotension. Choosing a sedative option at night (e.g. amitriptyline or pizotifen) may be suitable for someone with insomnia.

Drugs such as propranolol and verapamil should not be prescribed for patients with a history of selfpoisoning. Similarly, topiramate should be avoided in patients with a history of suicidal ideation. 


\section{Botulinum toxin $A$}

In Australia, if a patient has chronic migraine but has failed to improve with three oral prophylactic medicines or could not tolerate them, they qualify for Pharmaceutical Benefit Scheme (PBS) subsidised onabotulinum toxin A (Botox) therapy. This is given by a neurologist. Contrary to popular belief, this treatment does not work by relaxing the scalp, face or neck muscles (although the latter can be of additional benefit in some patients), but rather it slowly improves the migraine frequency and severity by altering the neurotransmitters involved in migraine. This treatment requires 31 injections subcutaneously in the head and neck every three months. Overall this is well tolerated without drug interactions or systemic adverse effects. However, headache, neck weakness, redness at the injection sites and heaviness of the eyelids are possible adverse effects. ${ }^{16}$

\section{Monoclonal antibodies}

A new class of injectable prophylactic drugs targeting calcitonin gene-related peptide (CGRP) have emerged recently. These appear to be well tolerated and reduce migraine frequency. Erenumab is a CGRP-receptor antibody, while fremanezumab and galcanezumab target the CGRP ligand. They are given as monthly subcutaneous injections. ${ }^{17}$ At the time of writing, there is approval for erenumab, fremanezumab and galcanezumab by the Therapeutic Goods Administration but not listed for PBS use yet in Australia, so access to this class of drug remains limited due to cost.

\section{Conclusion}

Migraine management starts with a correct diagnosis. Treatment of acute attacks requires early and effective medicines. If simple analgesics are inadequate, triptan drugs may be more effective. Preventive strategies to reduce migraine attacks include lifestyle modifications, management of comorbidities, behavioural and physical therapies, and pharmacological treatments. The choice of prophylactic medicines is guided by potential interactions, adverse effects and patient comorbidities. Novel preventive therapies such as the anti-CGRP monoclonal antibodies are targeted therapies to consider.

Managing migraine with acute and preventive strategies for those significantly affected can reduce the disability and loss of function caused by this disease. $<$

Bronwyn Jenkins has received fees for education and advisory boards from Allergan, Lilly, Novartis and Teva.

\section{REFERENCES}

1. Steiner TJ, Stovner LJ, Vos T, Jensen R, Katsarava Z. Migraine is first cause of disability in under 50s: will health politicians now take notice? J Headache Pain 2018:19:17. https://doi.org/10.1186/s10194-018-0846-2

2. Dodick DW. Migraine. Lancet 2018;391:1315-30. https://doi.org/10.1016/S0140-6736(18)30478-1

3. Puledda F, Shields K. Non-pharmacological approaches for migraine. Neurotherapeutics 2018:15:336-45. https://doi.org/ 10.1007/s13311-018-0623-6

4. Mauskop A. Nonmedication, alternative and complementary treatments for migraine. Continuum (Mineapp Minn) 2012;18:796-806. https://doi.org/10.1212/ 01.con.0000418643.24408.40

5. Neurology. In: eTG complete [digital]. Melbourne: Therapeutic Guidelines Limited; 2020. www.tg.org.au [cited 2020 Sep 1]

6. Vargas BB. Acute treatment of migraine. Continuum (Minneap Minn) 2018;24:1032-51. https://doi.org/10.1212/ CON.0000000000000639

7. Becker WJ. Acute migraine treatment. Continuum (Minneap Minn) 2015;21:953-72. https://doi.org/10.1212/ CON.0000000000000192

8. Dodick D, Lipton RB, Martin V, Papademetriou V, Rosamond W, MaassenVanDenBrink A, et al.; Triptan Cardiovascular Safety Expert Panel. Consensus statement: cardiovascular safety profile of triptans (5- $\mathrm{HT}_{1 \mathrm{~B} / 1 \mathrm{D}}$ agonists) in the acute treatment of migraine. Headache 2004;44:414-25. https://doi.org/10.1111/j.1526-4610.2004.04078.x

9. Cameron C, Kelly S, Hsieh SC, Murphy M, Chen L, Kotb A, et al. Triptans in the acute treatment of migraine: a systematic review and network meta-analysis. Headache 2015;55 Suppl 4:221-35. https://doi.org/10.1111/head.12601
10. Ferrari MD, Roon KI, Lipton RB, Goadsby PJ. Oral triptans (serotonin 5-HT1B/1D agonists) in acute migraine treatment: a meta-analysis of 53 trials. Lancet 2001;358:1668-75. https://doi.org/10.1016/S0140-6736(01)06711-3

11. Orlova Y, Rizzoli P, Loder E. Association of coprescription of triptan antimigraine drugs and selective serotonin reuptake inhibitor or selective norepinephrine reuptake inhibitor antidepressants with serotonin syndrome. JAMA Neurol 2018;75:566-72. https://doi.org/10.1001/ jamaneurol.2017.5144

12. Athavale A, Athavale T, Roberts DM. Antiemetic drugs: what to prescribe and when. Aust Prescr 2020;43:49-56. https://doi.org/10.18773/austprescr.2020.011

13. MacGregor EA. Migraine management during menstruation and menopause. Continuum (Minneap Minn) 2015;21:990-1003. https://doi.org/10.1212/CON.0000000000000196

14. Silberstein SD. Preventive migraine treatment. Continuum (Minneap Minn) 2015;21:973-89. https://doi.org/10.1212/ CON.0000000000000199

15. Schwedt TJ. Preventive therapy of migraine. Continuum (Minneap Minn) 2018;24:1052-65. https://doi.org/10.1212/ CON.0000000000000635

16. Dodick DW, Turkel CC, DeGryse RE, Aurora SK, Silberstein SD Lipton RB, et al.; PREEMPT Chronic Migraine Study Group. OnabotulinumtoxinA for treatment of chronic migraine: pooled results from the double-blind, randomized, placebocontrolled phases of the PREEMPT clinical program. Headache 2010;50:921-36. https://doi.org/10.1111/j.1526-4610.2010.01678.x

17. Khan S, Olesen A, Ashina M. CGRP, a target for preventative therapy in migraine and cluster headache: systematic review of clinical data. Cephalalgia 2019;39:374-89. https://doi.org/ $10.1177 / 0333102417741297$ 